\title{
Gloss Measurement Using Psychological Visual Characteristics
}

\author{
Seiichi Serikawa, ${ }^{\mathrm{a}, *}$ \\ ${ }^{a}$ Kyushu Institute of Technology, 1-1 Sensui, Tobata, Kitakyushu, Fukuoka, 804-8550, Japan \\ *Corresponding Author: serikawa@else.kyutech.ac.jp
}

\begin{abstract}
With the development of technology and the expansion of productions scales, the improvement of the product quality has become a more important issue than that of the quantity. Because human senses about gloss, glare, and image-sharpness highly affect the quality of the product, equation-based definitions which integrate the physical fitness qualities of a product are commonly used to describe such indexes. Accordingly, calculation using these equations may show results which are quite different from the perception of human beings. Therefore, in this study, we propose a new gloss measurement method using visual characteristics. Simulation results show that the glossiness obtained through this new method agrees well with the psychological sense of human beings.

Three issues are stressed in this speech as follows: (1) The problem with the conventional methods - glossiness measured does not agree with humans' psychological sense; (2) Explanation on how to quantify glossiness from the perspective of psychological sense; (3) Focus is given to a new gloss measurement method. The fitness of psychophysical glossiness is largely improved when visual characteristics are taken into account. A simplified model is also introduced to better clarify the mechanism of human beings' gloss perception.
\end{abstract}

